\title{
PERSPECTIVAS METODOLÓGICAS DE LA INVESTIGACIÓN EN SALUD PÚBLICA, BREVE MIRADA
}

\author{
Gilberto Bastidas¹,a, Tadeo Medina ${ }^{1, b}$, María Báez $^{1, c}$, María Antoima $^{1, d}$, Daniel Bastidas ${ }^{2, e}$
}

\begin{abstract}
RESUMEN
La investigación permite el fortalecimiento de las disciplinas, particularmente aquellas que se nutren de información de varias especialidades, como la salud pública. El objetivo de este informe es proporcionar de forma consolidada perspectivas metodológicas viables para enriquecer a la salud pública como ciencia. De la revisión destacan tres elementos claves: el tipo de abordaje investigativo, los enfoques de evidencia y competencia en el contexto metodológico de la indagación en la salud colectiva. Se concluye que el conocimiento sistemático, específico, integrado al contexto y obtenido en atención a las circunstancias de la investigación, el objeto de estudio y la ubicación institucional, contribuyen con el fortalecimiento de la salud pública como ciencia multidisciplinaria, directamente implicada, por su competencia y base en la evidencia, en la toma de decisiones en materia de salud por parte del Estado en áreas tan importantes como las necesidades, los servicios y los sistemas de salud.
\end{abstract}

Palabras claves: Conocimiento; Salud colectiva; Metodología (fuente: DeCS BIREME).

\section{METHODOLOGICAL PERSPECTIVES OF PUBLIC HEALTH RESEARCH. A BRIEF LOOK}

\begin{abstract}
Research allows for the strengthening of disciplines, particularly those that draw on information from various specialties, such as public health. The objective of this report is to provide consolidated methodological perspectives to enrich public health as a science. The review highlights three key elements: the type of investigative approach, the evidence and competence approaches in the methodological context of the inquiry into collective health. It is concluded that the systematic, specific knowledge, integrated to the context and obtained in attention to the circumstances of the investigation, the object of study and the institutional location, contribute with the strengthening of public health as a multidisciplinary science, directly involved, for its competence and based on the evidence, in the decision making in matters of health by the State in areas as important as needs, services and health systems.
\end{abstract}

Keywords: Knowledge; Public health; Methodology (source: MeSH NLM).

\section{INTRODUCCIÓN}

El conocimiento sistemáticamente organizado, producto de conceptos, definiciones y proposiciones derivadas de la prueba de elementos y variables en la medición de un fenómeno, constituye la base de la ciencia como formadora de teorías, es por ello, que la investigación es crucial en el fortalecimiento de las disciplinas como conocimiento riguroso y sistemático de un tema, especialmente en aquellas que se nutren de información de varias especialidades, como la salud pública, donde las iniciativas y competencias no pueden responder a la intuición, repetición y empirismo, sino al conocimiento derivado de un marco conceptual y metodológico sólido ${ }^{(1-5)}$.
La toma de decisiones en salud pública, resulta particularmente influenciada en países en desarrollo, por el empirismo e incluso por los vaivenes de los momentos políticos que atraviesan estas poblaciones, por tanto, la evidencia obtenida con procedimientos metodológicos reproducibles es la vía expedita para favorecer la toma de decisiones con apego a la realidad, así como en la perspectiva del creciente reconocimiento como ciencia formal a que aspira la salud pública, en este orden de ideas cobra importancia el objeto de este escrito, que no es otro que la descripción y análisis de perspectivas metodológicas de la investigación aplicables en salud pública ${ }^{(5,6)}$.

La correcta forma de investigar, si esto puede afirmarse, garantizaría a la salud pública su importante e indelegable

\footnotetext{
Departamento de Salud Pública, Universidad de Carabobo. Carabobo, Venezuela

Escuela de Medicina, Universidad de Carabobo. Carabobo, Venezuela.

a Médico cirujano, doctor en Parasitología; ${ }^{\mathrm{b}}$ médico cirujano, doctor en Ciencias Sociales; ${ }^{\mathrm{c}}$ licenciada en Educación, master en Investigación Educativa; ${ }^{\mathrm{d}}$ licenciada en Educación; ${ }^{\mathrm{e}}$ estudiante de Medicina

Recibido: 28/12/2017 Aprobado: 25/04/2018 En línea: 20/06/2018
}

Citar como: Bastidas G, Medina T, Báez M, Antoima M, Bastidas D. Perspectivas metodológicas de la investigación en salud pública, breve mirada. Rev Peru Med Exp Salud Publica. 2018;35(2):317-20. doi: 10.17843/rpmesp.2018.352.3352. 
papel en el aseguramiento de la salud como derecho humano fundamental, elemento necesario para el logro del mayor grado de bienestar ciudadano con especial hincapié en la dimensión colectiva y en las intervenciones de tipo inter y transectoriales, toda vez que la indagación permitirá la verificación constante de las teorías incorporadas a las prácticas profesionales y sociales de la salud pública, entonces se pretende con este escrito mostrar las herramientas metodológicas para facilitar la comprensión del continuo salud enfermedad dentro de la dinámica del contexto en que este ocurre ${ }^{(7-10)}$.

\section{ABORDAJE METODOLÓGICO}

Se realizó una revisión documental de la literatura disponible, de diversos estudios donde se reseñan y discuten tipos, modelos o clasificaciones de perspectivas metodológicas y de variables de investigación en la salud pública, empleando bases de datos físicas (bibliotecas) y virtuales (Bireme/ OPS, Medline, PudMed, Scielo, Google Académico) a partir de descriptores o palabras claves relacionados con constructos o propuestas, nombre de autores y de teorías o modelos. Los descriptores empleados fueron: perspectivas metodológicas, habilidades investigativas, modelos de investigación y teorías metodológicas. Para recuperar la literatura existente se emplearon los operadores boléanos AND y OR. La búsqueda fue filtrada para revisiones sistemáticas, editoriales, cartas al editor, ensayos y trabajos originales de investigación.

Fueron en total 138 los documentos resultantes incluidos según la temática (los directamente relacionados con los tipos de investigación en salud pública). Luego del análisis de las ideas y de los aspectos más relevantes para el tema de estudio, los artículos seleccionados, 19 en definitiva, fueron agrupados en tres aspectos básicos del conocimiento sobre la investigación en la disciplina, el primero alude a los elementos a considerar para la investigación en salud pública; el segundo muestra la influencia de la medicina basada en la evidencia en la investigación en salud pública; y finalmente se discute la competencia en la investigación en salud pública. Los documentos excluidos fueron aquellos repetidos en los motores de búsqueda, así como también, los que no tuvieron conclusiones claras o carecían de originalidad.

\section{LA INVESTIGACIÓN EN SALUD PÚBLICA}

Son tres los elementos que deben considerarse en la investigación en salud pública, el primero: su ubicación en el contexto general de la investigación en salud, el segundo: el campo y objeto de estudio con inclusión de las disciplinas que contribuyen con ella y el tercero: la ubicación institucional donde se enmarcará, en relación con las condiciones de salud (procesos biológicos, psicológicos y sociales) de los grupos poblacionales y de las acciones que la sociedad realiza en procura de mejorarla. En consecuencia, y como derivación del cruce que, sobre estas dimensiones realizara Frenk ${ }^{(11)}$ surgen cuatro áreas básicas de investigación: biomédica (condiciones y procesos determinantes de la salud/enfermedad), clínica (respuestas terapéuticas, preventivas o de rehabilitación), en necesidades de salud y en sistemas de salud.

Igualmente y por ser común en países de medianos y bajos ingresos económicos (los que mayoritariamente conforman el mundo) la escasez de recursos disponibles para satisfacer la creciente demanda de servicios sanitarios (elemento operativo del sistema de salud), se hace necesaria la investigación en prioridades o de financiación selectiva según la disponibilidad económica del sistema de salud, estudios que deben considerar, entre otros factores la evaluación económica como instrumento clave para la toma de decisiones, a partir, por ejemplo de la comparación de distintas alternativas de actuación en términos de costo y efecto sobre la salud ${ }^{(12)}$. Véase entonces que corresponde a la salud pública la generación de conocimiento en áreas tan grandes como las necesidades, los servicios y los sistemas de salud, esto por el carácter poblacional que parece distinguir a la investigación en salud pública de la biomédica y la clínica, estas últimas con enfoque claramente individual (11-14).

Por supuesto, el campo de investigación en salud pública resulta realmente amplio, por tanto, se plantea mayor especificidad sin llegar a la superespecialización en áreas sustantivas del conocimiento en materia de salud pública, que incluya como elemento clave el periodo o nivel en que se realizará la investigación. Por ejemplo, la medición de las necesidades de salud en la etapa inicial implica identificar las condiciones de salud y enfermedad que requiere atención y recursos; y en la etapa final debe verificarse la necesidad satisfecha en virtud de los recursos dispuestos para cubrirlas. Entonces, puede intentar adentrarse en lo complejo de las prácticas sanitarias, con enfoques específicos, pero no explícitamente divorciados del contexto integral y universal que define el campo de conocimiento científico de la salud pública ${ }^{(11,15)}$.

El objeto de investigación de la salud pública, su área sustantiva, versa sobre las necesidades sociosanitarias en relación con la salud positiva (índices de salud, proceso de desarrollo biopsíquico, condiciones no mórbidas que requieren atención), riesgos para la salud en lo económico, ambientales y otros, daños a la salud (respecto a morbilidad, incapacidad física y mental, y mortalidad), factores mediadores entre necesidades y servicios (equidad, tecnología y calidad de atención), utilización de servicios, factores mediadores entre servicios y recursos como productividad y accesibilidad, disponibilidad de recursos, y políticas de salud, con base en primer lugar, en 
las disciplinas básicas que contribuyen a la investigación en salud pública (la epidemiología, la demografía, las ciencias de la administración, la economía, la sociología, el derecho y la ética) y en segundo lugar con el alto grado de flexibilidad conceptual que la definen ${ }^{(11,16)}$.

\section{HACIA LA SALUD PÚBLICA BASADA EN EVIDENCIA}

La toma de decisiones clínicas con pruebas procedentes de investigaciones publicadas, método introducido en el ámbito del conocimiento por Guyatt ${ }^{(17)}$, como «Medicina basada en la evidencia», puede y de hecho se ha extrapolado al ámbito de la salud pública, como se certifica con la puesta en escena por Muir-Gray ${ }^{(18)}$ de la «Atención sanitaria basada en la evidencia», esto en respuesta al creciente apuro por proporcionar modelos de abordaje a los problemas sociosanitarios, de comprobar el resultado de las intervenciones en salud pública y de fortalecer su estructura teórica ${ }^{(19-21)}$.

La «Salud pública basada en la evidencia» parece ser la herramienta que fortalecería su fundamento teórico, toda vez que implica según Jenicek (22) «El uso consciente, explícito y juicioso de la mejor evidencia en la toma de decisiones sobre la atención a comunidades y poblaciones en el campo de la protección de la salud, la prevención de la enfermedad y el mantenimiento y mejora de la salud », por ese motivo la toma de decisiones en el sector salud se vería impelida con información real, con lo cual puede evitarse las improvisaciones, tan frecuentemente formuladas.

Ahora bien, la salud pública basada en la evidencia se ve dificultada fundamentalmente por la insuficiente tradición y formación metodológica del profesional de este campo, por su complejidad, por nutrirse de varias disciplinas y por su impacto multisectorial, a los que se suma, por supuesto, la pobre inversión estatal para investigar en salud pública, por tanto, el cuerpo de conocimiento en la materia no muestra la robustez necesaria para que la solución de los problemas sociosanitarios puedan basarse en la evidencia ${ }^{(17,20-21,23)}$.

\section{INVESTIGACIÓN PARA LA COMPETENCIA}

La globalización, el crecimiento de las economías, especialmente la de mercados, y la reducción de la intervención del Estado en los sistemas de salud, son fenómenos sociales que caracterizan a la comunidad mundial, con sus diferencias, matices y excepciones; por ejemplo, en Venezuela el Estado retoma su control e influencia sobre el sistema de salud, se cree sea, con el firme propósito de rebasar la fragmentación o segmentación de los sistemas de salud que resultan en muchos casos de reformas inconclusas y de visiones parciales de la situación sanitaria, hecho que genera transformaciones en el sector salud, que sin duda requiere ser investigados por la salud pública $^{(24-27)}$.

La realidad sanitaria, social, económica y política de un país, obliga al desarrollo de una salud pública competente, con base en la investigación, ya que, como señalara Suárez ${ }^{(25)}$ «(...) las competencias no son inmutables sino dialécticas, y se expresan en el desempeño, del cual se nutren, conformando una unidad epistemológica que -en consecuencia- varía en su dimensión espacial y temporal», entonces la identidad propia se constituye en el referente para determinar lo que se espera de la salud pública, con el firme propósito de mejorar el desempeño de los sistemas de salud global ${ }^{(28)}$.

Véase que la salud pública sustentada en competencias esenciales determinadas como producto de la investigación, supone una intervención exitosa, porque incluye la responsabilidad de asegurar la calidad en la atención de la salud, derivada de la clara definición de su papel, de sus funciones esenciales y de la operacionalización de los conceptos que la definen, por tanto, la efectiva inclusión de la salud pública en la agenda que procura el desarrollo de los países, es absolutamente indispensable, y pasa decididamente por clara definición de sus competencias, por supuesto, a través de la investigación (29-31).

Es aquí que tiene cabida la investigación sobre competencias esenciales para reafirmar la identidad propia de la salud pública, es decir, de las capacidades necesarias para el ejercicio de sus funciones cardinales en cualquier ámbito de la práctica sociosanitaria, con el objeto de ir más allá de las meras prácticas relacionadas con profesionales o roles, pues parece el camino a seguir en procura de una disciplina flexible y adaptable a distintos contextos, como única garantía para identificar, interpretar, interpolar, inventar y resolver problemas de la salud colectiva con relativa idoneidad y capaz de soportar el escrutinio metodológico más severo ${ }^{(24,30,31)}$.

Finalmente, se concluye que el asiento en el contexto general, el campo y objeto de estudio, y la ubicación institucional son los elementos que deben considerarse en la investigación en salud pública en las áreas biomédica, clínica, de necesidades de salud y de sistemas de salud, con base en el periodo o nivel en que se realiza el estudio, en la evidencia y en las competencias esenciales para reafirmar la identidad propia de la salud pública en el ámbito de la práctica sociosanitaria.

Conflicto de interés: Los autores señalan que no existen conflictos de interés.

Financiación: Los autores señalan que no la hubo. 


\section{REFERENCIAS BIBLIOGRÁFICAS}

1. Cabrera G. Uso de teorías y modelos en artículos de una revista latinoamericana de salud pública, 2000-2004. Rev Saúde Pública. 2007;41(6):963-69.

2. Van-ryn M, Heany C. What's the use of theory. Health Educ Quart. 1992;19:315-30.

3. Ulin P, Robinson E, Tolley E. Investigación aplicada en salud pública: Métodos cualitativos [Internet]. Washington, D.C: OPS; 2006. [Citado $15 \mathrm{de}$ enero de 2018]. Disponible en: http:// apps.who.int/iris/bitstream/handle/10665/166144/9275316147.pdf;jsessionid=031E1E $5227 \mathrm{C} 3 \mathrm{~F} 4 \mathrm{CC}$ FBCE7507CDF3DDA? sequence $=1$

4. Cantú P. Investigaciones en salud pública. Consideraciones y procesos. Culcyt. 2008;5(28):29-36.

5. Cabrera, G. Teorías y modelos en la salud pública del siglo XX. Colomb Méd. 2004;35(3):184-8.

6. Tancred T, Schleiff M, Peters D, Balabanova D. Health policy and systems research training: global status and recommendations for action. Bull World Health Organ. 2016; 94(7):491500. doi: 10.2471/BLT.15.162818.

7. Organización Panamericana de la Salud. Promoción de la salud: una antología [Internet]. Washington DC: OPS; 1996. [Citado 15 de enero de 2018]. Disponible en: http://iris.paho.org/xmlui/handle/123456789/3318

8. Aristizabal G, Blanco D, Sánchez A, Ostiguin R. El modelo de promoción de la salud de Nola Pender: Una reflexión en torno a su comprensión. Enferm univ. 2011;8(4):16-23.

9. Organización Panamericana de la Salud. La salud pública en las Américas: Nuevos conceptos, análisis del desempeño y bases para la acción [Internet]. Washington DC: OPS; 2002. [citado el 10 de diciembre de 2017]. Disponible en: http://wwwl.paho. org/hq/dmdocuments/2010/FESP_ Salud_Publica_en_las_Americas.pdf

10. Gerhardus A, Becher H, Groenewegen P, Mansmann U, Meyer T, Pfaff $\mathrm{H}$, et al. Applying for, reviewing and funding public health research in Germany and beyond. Health Res Policy Syst. 2016;14(1):43. doi: 10.1186/s12961-016-0112-5.

11. Frenk J, Bobadilla J, Sepúlveda J, Rosenthal J, Ruelas E. Un modelo conceptual para la investigación en salud pública. Bol of Sanit Panam. 1986; 101(5):477-92.
12. García-Altés A, Navas E, Soriano M. Evaluación económica de intervenciones de salud pública. Gac Sanit. 2011;25(Supl 1):25-31. doi: 10.1016/S0213-9111(11)70005-X.

13. Bachelard G. La Formación del Espíritu Científico [Internet]. Buenos Aires: Siglo XXI; 2000. [Citado el 10 de diciembre de 2017]. Disponible en: https:// doctoradousbcienciaseducacion.files. wordpress.com/2013/01/bachelard-laformacion-del-espiritu-cientifico.pdf

14. Donabedian, A. Aspects of Medical Care Administration: Specifying Requirements for Health Care. Massachusetts: Harvard University Press; 1973.

15. Degeling C, Carter S, Rychetnik L. Which public and why deliberate? A scoping review of public deliberation in public health and health policy research. Soc Sci Med. 2015;131:114-21. doi: 10.1016/j.socscimed.2015.03.009.

16. Guével M, Pommier J. Mixed methods research in public health: issues and illustration. Sante Publique 2012;24(1):23-38.

17. Guyatt, G. Evidence-based medicine. ACP J Club. 1991;114:A16. doi:10.7326/ ACPJC-1991-114-2-A16.

18. Cediel-Becerra N, Krause G. Herramientas para la toma de decisiones en salud pública basadas en la evidencia y priorización de enfermedades. Rev Salud Pública. 2013;15(5):694-706.

19. Franco A. Tendencias y teorías en salud pública. Rev Fac Nac Salud Pública. 2006 24(2):119-130.

20. Nebot M. Evaluación en salud pública: ¿todo vale? [editorial]. Gac Sanit. 2007; 21:95-6.

21. López J, Bonfill X. Sobre la Salud Pública basada en pruebas. Rev Esp Salud Pública [Internet]. 2008;82(1):1-4.

22. Jenicek M. Epidemiology, evidencedbased medicine, and evidence-based public health. J Epidemiol. 1997;7:187-97.

23. Victora C, Habicht J, Bryce J. Evidencebased public health: moving beyond randomized trials. Am J Public Health. 2004;94:400-5.

24. Programa de las Naciones Unidas para el Desarrollo (PNUD). Informe sobre desarrollo humano. Los objetivos de desarrollo del milenio: Un pacto entre las naciones para eliminar la pobreza [Internet]. Nueva York: PNUD-Grupo Mundi Prensa; 2003. [Citado el 20 de septiembre de 2017]. Disponible en: http://hdr.undp.org/sites/default/files/ hdr_2003_es.pdf

25. Organización Panamericana de la Salud. Informe de la salud en las Américas [Internet]. Washington, D.C: OPS; 2007. [Citado el 20 de septiembre de 2017]. Disponible en: http://www.paho.org/hia/ archivosvol1/volregionalesp/SEA07\%20 Regional\%20SPA\%20 Cap\%201.pdf

26. Organización Panamericana de la Salud. Programa de líderes en salud internacional [Internet]. Washington, D.C: OPS; 2013. [Citado el 26 de octubre de 2017]. Disponible en: https://www.paho.org/hq/index. php?option $=$ com content\&view $=$ article\&id=12754\&Itemid=3499\&lang=es

27. Organización Panamericana de la Salud. Competencias esenciales en salud pública: un marco regional para las Américas [Internet]. Washington, D.C: OPS; 2013. [Citado el 30 de enero de 2018]. Disponible en: https://cursos.campusvirtualsp.org/pluginfile.php/72114/mod_label/ intro/competencias-SPA\%20final.pdf

28. Davó M, Gil-González D, Vives-Cases C, Álvarez-Dardet C, Ronda E, Ortiz-Moncada Razón, et al. ¿Quiénes y qué pueden hacer en salud pública? Las competencias profesionales como base para la elaboración de programas en el Espacio Europeo de Educación Superior. Gac Sanit. 2009;23(1):5-12.

29. Muñoz F, López-Acuma $D$, Halverson $P$ Guerrero C, Hanna W, Larrieu M, et al. Las funciones esenciales de la salud pública: Un tema emergente en las reformas del sector de la salud. Rev Panam Salud Publica. $2000 ; 8(1 / 2): 126-34$

30. Tobón S. Formación integral y competencias. Pensamiento complejo, currículo, didáctica y evaluación. Bogotá: Ecoe; 2010.

31. Lafferty N,Manca A. Perspectives on social media in and as research: A synthetic review. Int Rev Psychiatry.2015;27(2):85-96. doi: 10.3109/09540261

\section{Correspondencia: Gilberto Bastidas}

Dirección: Urbanización Terrazas de San Diego, municipio San Diego, estado Carabobo, Venezuela.

Teléfono: +584247789944

Correoelectrónico:bastidasprotozoo@hotmail.com 